\title{
ON THE ETIOLOGY OF SCARLET FEVER. ${ }^{1}$
}

By W. MaIr, Research Pathologist to the Metropolitan Asylums Board, London.

From the Labaratory of the Metropolitan Asylums Board, Lister Institute, London, S.W.

IN January of last year, in a paper in this Journal on "Experimental Scarlet Fever in the Monkey " (19154), I gave a preliminary description of a diplococcus isolated from the throats of scarlet fever patients, showed that it produced in monkeys a disease resembling scarlet fever in several respects, and suggested for it the name Diplococeus scarlatinoe.

The object of the present communication is to give a fuller description of this diplococcus, with the technique used for its isolation and identification, and to adduce additional evidence in favour of its causal relationship to the disease.

Morphology, Cultural Characters, and Conditions of Growth.

Throughout this paper the terms "ordinary broth" and "ordinary agar" refer to nutrient media prepared in the usual way from meat extract and peptone. The peptone used is either Witte's or another brand prepared from fibrin and is sugar free. Except where otherwise indicated the meat extract is prepared from ox's heart. The reaction of the media is faintly alkaline to litmus. "Serum broth" and "serum agar" indicate the same media with the addition of 5 to 10 per cent. of horse serum. The serum is sterilised by filtration and is added to the broth or melted agar by means of a pipette, the quantity being roughly estimated by the number of drops.

In its most typical form, in cultures on serum agar or in serum broth not more than twenty-four hours old, the Diplococeus scarlatince is an oval or oat-shaped coccus measuring about $1.2 \mu$ by $0.6 \mu$. It is arranged for the most part in pairs, end to end, and both ends of each member of the pair are distinctly pointed. Shorter and rounder forms are common, measuring about $1 \mu$ by $0.8 \mu$. Short chains up to about ten pairs also occur in serum broth. It is non-motile and retains the

${ }^{1}$ Received February 22, 1916. 
stain in Gram's method. Capsules cannot as a rule be demonstrated in cultures, but occasionally, when stained by the Hiss copper-sulphate method, capsules can be made out. Occasionally, also, distinct capsules can be demonstrated by this method in the blood of mice; not infrequently poorly developed capsules are seen. The diplococcus is therefore closely related to the Diplococcus pneumonice. The optimum temperature for growth is about $36^{\circ}$ to $37^{\circ} \mathrm{C}$, and on suitable media growth occurs at this temperature equally well under aerobic and anaerobic conditions. No growth occurs above $40^{\circ} \mathrm{C}$. or below $20^{\circ} \mathrm{C}$. Growth is not easily obtained on ordinary media even at $37^{\circ} \mathrm{C}$. When growth occurs in ordinary broth, it is characterised by a faint diffuse cloudiness. Most strains refuse to grow in ordinary broth or on agar even after prolonged cultivation. The diplococcus can be grown on glucose agar and in glucose broth. If calcium carbonate be added to the glucose broth to neutralise the acid formed, copious growth is obtained, and for certain purposes this forms the best medium for its cultivation. The addition of from 5 to $10 \mathrm{per}$ cent. of horse serum or defibrinated rabbit blood to ordinary agar or broth gives the media which are most generally useful.

On serum agar the colonies are at first minute and transparent, later they become larger and more opaque. There is nothing specially characteristic about them except that they frequently present an elevated margin with depressed centre, giving the ringed appearance often found in pneumococcus cultures. On blood-agar plates prepared with fresh defibrinated rabbit's blood, thinly sown on the surface and incubated in a metal box to prevent evaporation, the colonies after eighteen hours are large, measuring 3 to $6 \mathrm{~mm}$. in diameter, distinctly raised above the surface of the medium, and of soft consistence. By reflected light they appear white, by transmitted light, green. These large colonies show no halo; smaller colonies may show a narrow, green, translucent zone around them. The clear, colourless, halo characteristic of the so-called hæmolytic streptococci is never seen. In serum brotb a uniform turbidity is produced; if the growth is copious a slight deposit forms at the bottom of the tube, which on gentle shaking can be distributed quite uniformly. In undiluted horse or rabbit serum uniform diffuse growth also occurs.

The diplococcus grows readily in milk with acid formation. Clotting only occurs after two or three days at $37^{\circ} \mathrm{C}$.

\section{Fermentation Reactions.}

These were carried out in litmus peptone water containing 2 per cent. peptone and 1 per cent. of the substance to be tested with the addition of a few drops of horse serum to each tube. The diplococcus produces enough acid to turn the litmus distinctly red in two days at $37^{\circ} \mathrm{C}$. in the following: glucose, mannose, galactose, lævulose, maltose, 
lactose, saccharose, raffinose, and dextrin. Inulin and salicin are also fermented, but with some strains longer time is required. Dulcite and sorbite are not attacked. Mannite is slowly fermented by some strains, but not by all. A slight degree of acidity is produced in arabinose and xylose, none in isodulcite. Glycol is slowly fermented, but neither glycerol nor erythrite is attacked.

\section{Bile SaLt Test.}

If to a serum broth culture enough sodium taurocholate be added to make the concentration about 1 per cent., the diplococcus undergoes complete lysis in a few minutes. The bile salt is added in the form of a 10 per cent. solution in normal saline. If the growth is abundant and not more than one day old, the reaction is clearly visible to the naked eye. After about five minutes a diffuse turbidity begins to appear, which is apparently only partly due to the precipitation of insoluble bile acids, as it does not clear up entirely on the addition of alkali. The tubes must, therefore, be kept under observation from the time the bile salt is added, in order that the reaction may not be missed. Under certain conditions, for example, in old cultures or in broth which contains appreciable amounts of sugar, the bile acid precipitation may occur before the bacteriolysis is complete, and the reaction may thus be obscured. A comparison of stained films, made before and after the addition of the bile salt, must be made in any case of doubt. If the meat extract has been prepared from leg of beef instead of from heart muscle, the cultures are often so acid that an immediate precipitate occurs on the addition of the bile salt. If this precipitate is dissolved by the careful addition of the requisite amount of alkali, the reaction can still be observed by the naked eye.

This reaction can also be obtained with emulsions in normal saline made from cultures on serum or blood agar. Boiling these emulsions, or treating them with cbloroform or caustic alkali, prevents the reaction. The addition of 10 per cent. N/10 caustic soda causes a distinct clearing of the emulsion, but on microscopical examination the diplococei are seen to be quite well preserved, and the subsequent addition of bile salt causes no lysis.

Neufeld $\left(1912^{5}\right)$, who was the first to describe this phenomenon of the lysis of "pneumococei" by bile salts, insists on the necessity for using fully virulent cultures for the test. In the case of the Diplococcus scarlatince it apparently does not matter how long the strain nsed has been grown on artificial media, and the reaction can be obtained with cultures which are several days old. This statement applies also to all other strains of pneumococei which are dealt with in this paper, and the reaction, as described above, gives a very easy and certain means of distinguishing members of this group from streptococci which are not soluble in bile. 


\section{Pathogenicity for animals.}

Mice.-The pathogenicity of the Diplococcus scarlatince for mice is of a low order compared with that of the Diplococcus pneumonice. With most strains it is possible to raise the virulence by passage until 0.02 c.c. of an eighteen hours' serum broth culture kills within twenty-four hours on subcutaneous injection. The lethal dose when given intraperitoneally is not less. This is about the limit of virulence which can be attained with certainty, but doses of 0.001 c.c. occasionally prove fatal after two or three days. With some strains it has not been found possible to raise the virulence beyond fatal doses of 0.1 to 0.25 c.c. As the cultures show as a rule about 200 million diplococci per c.c., the fatal dose is of the order of a million, a very different result from that obtained with highly virulent pneumococci.

The virulence, although of a low order, is fairly well maintained in subcultures, and there is never any difficulty in again raising the virulence of a laboratory strain. In blood films from the dead mice, the diplococcus can generally be found, but only occasionally in very large numbers. In its typical form it appears in pairs of oat-shaped cocci, similar to those seen in cultures, but often slightly larger, measuring $1.5 \mu$ by $0.8 \mu$. These long forms are usually accompanied by shorter, rounder, and less characteristic forms $(1 \mu \times 0.8 \mu)$, and some strains show only the shorter type. Not infrequently capsules can be demonstrated by the Hiss copper-sulphate method, but these are seldom well developed.

The Diplococcus scarlatine is also pathogenic to mice when they are fed with cultures or with other mice dead from the infection. After such feeding experiments, some of the mice die and the diplococcus can be recovered from their blood. This statement applies also to wild mice, and in view of the cannibalistic habits of these animals, this observation may have an important bearing on the epidemiology of scarlet fever.

Rats.-When injected subcutaneously with large doses (2 to 5 c.c.) of virulent cultures, rats develop a septicæmia showing capsulated diplococci in their heart blood. There is generally marked odematous inflammation of the subcutaneous tissues.

Rabbits. - A fatal septicæmia may also be produced in rabbits by large doses $(2$ c.c.) of strains, the virulence of which has been raised by mouse passage. If the rabbit survives for several days, there is marked fibrinous inflammation in the subcutaneous tissues spreading from the site of injection. Sublethal doses often produce extensive cutaneous sloughs.

Adult guinea-pigs are comparatively insusceptible. Large doses (5 to 10 c.c.) on subcutaneous injection produce local fibrinous inflammation with necrosis of the skin and ulceration. Usually when this has occurred the animal dies after a week or ten days, and the 
diplococcus may be recovered from its blood, frequently accompanied by other bacteria.

Young guinea-pigs are more susceptible and may die rapidly of septiccemia.

Monkeys. - The reaction in the monkey to the subcutaneous injection of the Diplococcus scarlatince has been described by me in the paper already referred to, but it is necessary to amplify that description in the light of further experience. It was shown in the previous paper that the reaction consists in a febrile rise of temperature accompanied by certain changes in the cytoplasm of the polymorphonuclear leucocytes (Döhle's bodies), with the production of a marked local inflammation and, occasionally, sloughing of the skin or abscess formation. It was also stated that the diplococcus, on isolation from the throat of a patient (by the method then used), did not always possess sufficient virulence to produce this reaction in pure culture. It has now to be added that the virulence of the diplococcus for monkeys can be increased by passage through mice. After such passage a reaction can always be obtained in the monkey. A dose of 1 to 2 c.c. of a serum broth culture, of which 0.02 c.c. is lethal for mice, gives rise to a severe reaction in the monkey. If the injection be made on the inner side of the thigh, the local inflammation appears first as an odematous swelling of the loose tissues about the genitals. The temperature rises several degrees within twelve hours, and remains elevated while the local inflammation is acute. The inflammation spreads over both buttocks, and hæmorrhagic patches appear in the skin. These areas of skin slough and leave deep ulcers, exposing the underlying muscle. If the animal recovers, the ulcers heal rapidly by granulation and cicatrisation. There is a marked initial polymorphonuclear leucocytosis, generally succeeded by a leucopenia.' Döhle's bodies appear in the leucocytes and persist while the temperature is febrile, or longer where there is severe toxæmia, for in this case the temperature often falls and may even become subnormal. A considerable percentage of the leucocytes show the myelocytic type of nucleus. In a less severe infection there may be abscess formation, but no sloughing. The diplococcus is then found in the pus in pure culture. If the ulcers are extensive, death may occur in the second week or later, and the characteristic changes in the leucocytes persist to the end. The blood is then found either to be sterile or to contain only secondary invaders such as streptococci, staphylococci, or B. proteus; death is therefore due to a toxæmia, the secondary infection being probably, in most cases, a terminal phenomenon. The above-described reaction, with local sloughing or abscess formation, ending in recovery or toxæmic death in the second, third, or fourth week, is that which may be regarded as typical for the Diplococcus scarlatince. If, however, a large dose $(10$ c.c.) of a virulent culture be injected, the monkey may die within two or three days or at most a week, with septicæmia, the 
diplococcus being recovered from its blood. There is always, even in this case, marked local inflammation and evidence of profound toxæmia. With or without an initial rise, the temperature falls far below the normal, and the initial leucocytosis rapidly gives place to an intense leucopenia. The few leucocytes seen are often so profoundly altered that it is difficult or impossible to classify them. It is indeed doubtful if the presence of the diplococcus in the blood should be taken as indicating a true septicæmia; it may well be merely a terminal invasion in an animal already moribund from toxæmia.

The Reaction in the Monkey to other Pneumococci.

Pneumococci from other sources produce in the monkey a febrile reaction with the appearance of Döble's bodies in the leucocytes. In a moderately severe, but not immediately fatal, infection the clinical features may be quite different from those produced by the Diplococcus scarlatince, notably in the absence of severe local inflammation. Thus a pneumococcus ( $\mathrm{Pn}$. 40) from the sputum of a case of bronchopneumonia, caused in one monkey a fever, with Döhle's bodies, lasting for ten days with practically no reaction at the site of injection. Another monkey injected with the same pneumocoecus was found dead on the tenth day and showed pleurisy with fibrinous deposit and seropurulent exudate. It is possible that the immediate cause of death in this case was an abdominal injury on the ninth day. The pneumococcus was recovered from the pleura and from the heart blood. There was little local reaction at the site of injection. This pneumococcus was shown by the complement fixation test to be very far removed from the Diplococcus scarlatince. It may be stated, generally, that pneumococci of this type show more tendency to invade the blood stream and produce visceral lesions or general septicæmia than does the Diplococcus scarlatina. On the other hand, the reaction described in the monkey cannot be regarded as specific for the Diplococcus scarlatino. A pneumococcus ( $\mathrm{Pn}$. $\mathrm{R}$ ) isolated from the sputum of a case of pneumonia the day after the crisis gave a typical "scarlet fever" reaction in the monkey. This pneumococcus was shown by complement fixation tests to be much more closely related to the Diplococcus searlatince, but still quite distinguishable from it.

The fact appears to be that the production of Döhle's bodies in the monkey is characteristic of the whole pneumococcal group, and that the "scarlet fever" reaction is characteristic of a subgroup (possibly a large one) of which the Diplococcus scarlatince is the most typical member. The matter is obviously one which requires further investigation.

Immunity.-A considerable degree of immunity to the Diplococcus scarlatino is produced in monkeys either by a single moderately severe infection or by repeated doses increasing in amount and virulence. Immunity can also be established by injections of bacterial extracts, 
the preparation of which is described later in connection with complement fixation.

In one monkey the immunity produced by a single infection was tested a year later and was found to be such that an injection which was fatal to a control animal produced only a slight reaction.

Arthritis. - In two monkeys during the process of gradual immunisation, the occurrence of arthritis and tenosynovitis lasting for a few days and then clearing up completely, has been noted. In one of these the arthritis occurred on the day following the second injection, the first injection having been given seven days before the second. In the other monkey the arthritis occurred on the day following the fifth injection. In this case also the fourth injection had been given a week previously, the earlier injections having been made at intervals of from two to three weeks. A similar observation was recorded in one monkey (Rbesus No. 17) in my previous paper. In this case the arthritis occurred on the eighth day after a single injection.

It remains to add that $I$ have not so far succeeded in infecting monkeys by feeding or swabbing the throat either with pure cultures or with material from scarlet fever throats. The experiments with this object in view have not, however, been very numerous. A single feeding experiment in the case of a young chimpanzee gave a doubtful result. This experiment will be described in some detail, as, though inconclusive, $I$ am of opinion that it shows an infection of the chimpanzee by means of pure cultures.

On 9th February the chimpanzee "B" was fed with cultures, in milk, of three different strains of the diplococcus; from the 12 th to the 15 th a polymorpho-nuclear leucocytosis was noted, and a rise of temperature began on the $15 \mathrm{th}$, which lasted with intermissions until the 23rd. Döhle's bodies were not found. The temperature readings and the leucocyte counts are given in the following table:-

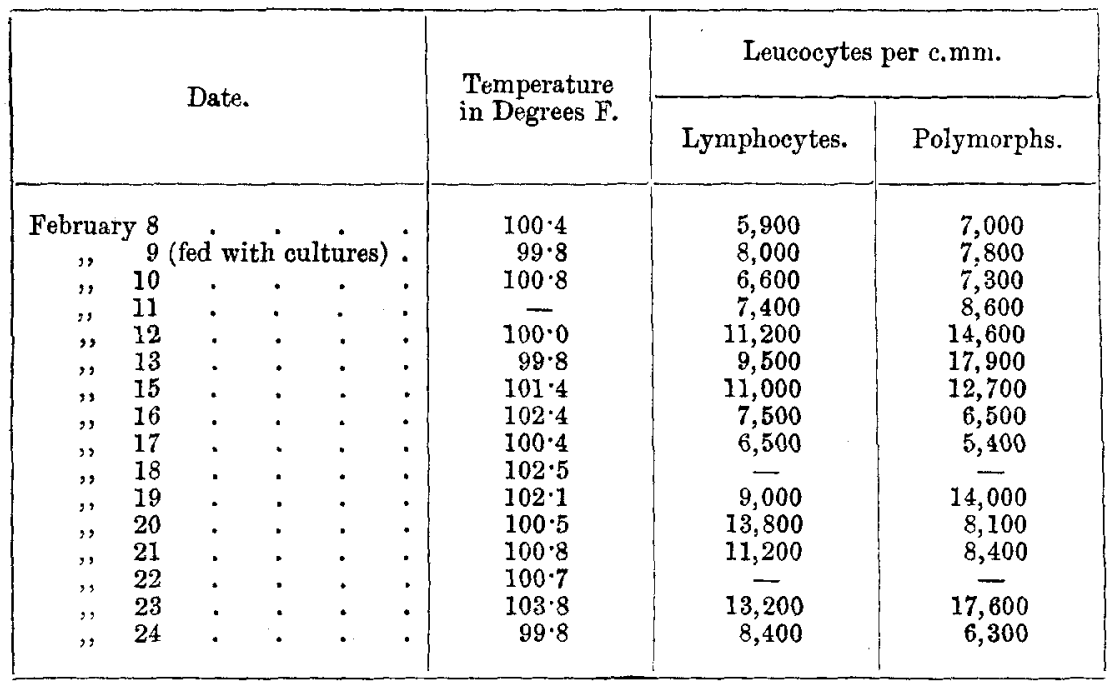


Unfortunately the throat was not examined owing to difficulty in handling the animal. There is, however, evidence that a throat lesion occurred, for on 30 th March a submandibular abscess was opened, and from the pus a streptococcus, but not the Diplococcus scarlatince, was obtained. This finding exactly corresponds to what occurs in post-scarlatinal cervical abscesses in the human subject. On 15th April the throat of the same chimpanzee was swabbed with a blood-agar culture of the Diplococcus scarlatino, made directly from the heart blood of the mouse used in isolating it from a case of scarlet fever. Six days later the diplococcus was recovered from the chimpanzee's throat, but no symptoms of any kind were observed. The suggestion is that the chimpanzee is susceptible to infection by pure cultures and that the slight reaction obtained on the first occasion was sufficient to protect against a second infection.

\section{IsOlation AND IDENTIFICATION OF DIPLOCOCCUS SCARLATINAE.}

Several different methods have been used for the isolation of the diplococcus; two of these were described in my previous paper, namely-

I. The injection of mouth washings or impure cultures into the subcutaneous tissues of the monkey and the withdrawal of fluid therefrom one or two days later;

II. Isolation directly from the throat by plating on serum agar and incubating the plates for tbree or four days in a closed box.

The methods since devised which have proved more satisfactory may be divided into two groups-
A. Isolation by purely cultural methods.
B. Isolation by mouse inoculation.

A (1).-A series of blood-agar plates is prepared, using from $\frac{1}{2}$ to $\frac{3}{4}$ c.c. of fresh defibrinated rabbit's blood for each plate. A swab taken from the tonsils of the patient is washed in sterile normal saline or broth, and a few loopfuls are placed on the first plate. The plates are then rubbed in succession with a glass rod, incubated in a closed box, and examined next day for characteristic colonies.

A (2).-The swab from the patient's throat is washed in a tube of serum broth, which is incubated overnight. Next day a series of rabbit-blood agar plates is sown from this tube, taking only a minute quantity for the first plate; the amount which adheres to a bent platinum wire is sufficient, and only two or three plates need be used. Comparative experiments show a much larger percentage of positive results by this method.

If for any reason it is desired to dispense with the use of mice, a combination of these two methods gives a fairly satisfactory technique for the isolation of the diplococcus.

B (1).- The swab washing is injected subcutaneously into a mouse. If the mouse dies within two days its heart blood is plated on rabbit-blood agar. This method has proved less satisfactory than 
B (2).-The swab is washed in a serum broth tube which is incubated overnight, care being taken to keep it in an upright position so that the streptococci may sediment at the bottom. Next day from 1 to 2 c.c. of the upper portion of the impure culture is injected subcutaneously into a mouse. The mouse usually dies within twenty-four hours. A little of its heart blood is then plated on two rabbit-blood agar plates. A preliminary microscopical examination of the blood gives information which is useful in determining the amount to be used for the plates. If the mouse has not. died after two days, but is obviously ill, it should be killed with chloroform. In this case as much blood as possible should be added to a serum-broth tube in addition to the smaller quantity used for the plates. The mice used vary from 10 to 20 grms. in weight, the most usual weight being 15 grms. This method is the most satisfactory of all, and has never failed to give a positive result where the presence of the diplococcus has been shown by any other method.

It is particularly to be noted with regard to methods A (2) and $B$ (2) that they only succeed as described if the broth used is practically free from sugar. Broth made from leg of beef instead of from heart muscle frequently contains so much sugar that the acidity developed by the mouth bacteria inhibits the growth of the diplococcus and may lead to a negative result. This difficulty may, however, be got over by adding a little calcium carbonate to the broth before the swab is washed in it.

By whatever method the rabbit-blood agar plates have been obtained, they are incubated at $37^{\circ} \mathrm{C}$. in a closed metal box to prevent drying, and are examined next day for characteristic colonies. The typical appearances described above are not always exactly reproduced; often instead of isolated colonies, or along with them, green streaks are found, and the white appearance by reflected light. is not present unless the growth is vigorous. In the absence of typical colonies, therefore, any green colony should be examined. A few subcultures are made in serum broth. After incubation these should show diffuse and not very abundant growth with little or no sediment. Any tube showing clear broth with a sediment may be discarded.

Subcultures are then made on serum agar, in ordinary broth, and in raffinose peptone water. The serum-broth tubes are tested for the bile salt reaction. A bile-soluble oat-shaped diplococcus which ferments raffinose, isolated in this way from a scarlet fever throat, may be regarded as the Diplococcus scarlatince. In the case of diplococci from other sources serological reactions are necessary to determine their relationship, and of these the fixation of complement test has so far been found the most satisfactory.

As will be seen later, diplococei which are very closely related to the Diplococcus scarlatinoe occur in a certain percentage of normal throats and in other diseases, and it is not yet possible accurately to classify these. 


\section{Statistical: Examinations of Scarlet Fever and Control} THROATS.

In a series of fifty cases of scarlet fever examined in the first. week by one or other, or several, of the methods above described, the Diplococcus scarlatinoe has been isolated from the throat in fortytwo, i.e., in 84 per cent. In the last twenty-four cases of the series, using method $B(2)$, positive results were obtained in twenty-one, i.e., in 87.5 per cent. Also using this method a series of examinations at later stages of the disease gave the following results:-

Of eleven cases in the third week the diplococeus was found in eight, of eleven cases in the fourth week in seven, and of eleven cases in the fifth week in two. In a series of eighteen cases an attempt was made to determine the length of time for which the diplococcus persists in the throat. In six of these (33 per cent.) it was found as late as the eighth week, in four (22 per cent.) in the eleventh week, and in one in the fourteenth week. The four cases in which the diplococcus persisted beyond the eighth week were kept in hospital for the purpose of the examinations. In the ordinary course they would have been discharged many weeks earlier. An attempt, without success, was made to get rid of the diplococcus by treatment with chlorine water, and the patients were finally discharged before two consecutive examinations with negative results were obtained. The above figures probably understate the persistence of the diplococcus, as they are to some extent dependent on the results of single negative examinations. It may, however, be safely concluded that in the majority of cases the diplococcus disappears from the throat about the fifth week, and that the occurrence of carrier convalescents is distinctly common.

It should be mentioned that the cases examined were of varying degrees of severity, several of them ending fatally, but no fulminant case is included in the series.

In a control series of thirty-five cases the throats were examined by the same technique-method B (2)-and bile-soluble pneumococci were found in twelve, or 34 per cent. The controls were for the most part normal, adults and children being included in about the same proportions as in the scarlet fever cases. Some of them were convalescents from various diseases. Two of the negative controls were cases of acute tonsillitis. Four (also negative) had been recently in contact with scarlet fever patients; the remainder had not been exposed to any known source of infection. None of the control pneumococei showed the typical characters of the Diplococcus scarlatino on rabbit-blood agar plates, but most of them came within the limits of variability shown by the latter. In fermentation reactions no constant differences could be detected; two of them fermented raffinose only slowly, and sometimes failed to do so. Minor differences in 
morphology were observed, both in cultures and in mouse blood; but by none of these characters could a definite distinction from the Diplococcus scarlatinoe be made. Eight of the strains were tested by complement fixation, and all showed a very close relationship to the Diplococcus scarlatino.

With the anti-scarlatinal sera available they could be differentiated from one group of the scarlatinal strains, but not from another. This matter will be referred to again under "complement fixation." In pathogenicity for mice, also, in so far as this was tested, the control strains did not differ from the scarlatinal. One strain, after several mouse passages, produced in a monkey a severe reaction ending in septicæmic death on the eighth day, here also showing no difference from the Diplococcus scarlatina.

It may be asked whether previous observations on the relative frequency of "pneumococci" in scarlet fever and in control throats are available. They exist, but they are not of use in the present connection on account of the ill-defined connotation of the term. "pneumococcus."

Thus Ruediger $\left(1906^{\circ}\right)$, in a study of the streptococei from scarlatinal and normal cases, found "pneumococci" in sixty-four out of seventy-one scarlatinal throats, and in forty-two out of fifty-one normal throats. In one scarlatinal case, which he regarded as exceptional, the pneumococcal colonies outnumbered the streptococcal, and this pneumococcus was fully virulent for rabbits. There is no doubt that in this case at least he had in hand the Diplococcus scarlatince. Ruediger's criteria for the identification of "pneumococci" were defective, as he relied chiefly on the fermentation of inulin and the appearance of the colonies on blood-agar plates. In my experience no diplococcus can be identified as a member of the pneumococcal group (apart from animal experiment) unless it is shown to undergo lysis in bile salt solution.

\section{Complement Fixation.}

Reference has been made to the possibility of distinguishing the Diplococcus scarlatince from other pneumococci by means of complement fixation. The detailed description of the work must be reserved for a future paper when it is more complete, but the technique used and the general nature of the results so far obtained may be shortly indicated here. Two monkeys were highly immunised to the Diplococcus scarlatince, in the one case four different strains being used, and in the other a single strain. The sera of these monkeys were used in the tests, practically identical results being obtained with both. For use as antigens bacterial extracts were prepared by growing the diplococcus in question in glucose broth with added calcium carbonate. After from three to five days' growth the broth culture was filtered through porcelain, the bacterial deposit on the filter was washed off, thoroughly emulsified, and made up to one-tenth of the original volume of the culture with normal saline containing 0.5 per cent. phenol. The thick 
emulsion thus obtained was frozen and as a rule allowed to stand in the freezing mixture overnight. It was then slowly thawed, warmed $\mathrm{up}$, and left in the incubator for a few hours, and centrifugalised. By this treatment pneumococci undergo very complete bacteriolysis, and little deposit (except chalk) is thrown down by the centrifuge. The extract is opalescent in appearance and syrupy in consistence. At the temperature of the cold room (about $2^{\circ} \mathrm{C}$.) it usually sets to a jelly. A nitrogen estimation of one sample indicated a protein content of about 7 per cent. These bacterial extracts maintain their properties for many months if kept in the cold room, and, as has already been mentioned, they can be used for the immunisation of animals.

As complement, fresh or salted guinea-pig serum was used. A remarkable fact has here to be noted. In a considerable proportion of guinea-pigs the serum contains pneumococcal antibodies, and the serum of these animals is of course useless as complement for work in this group. In young guinea-pigs, under 300 grms. in weight, the proportion in which the serum shows these antibodies is about 1 in 6 ; in older guinea-pigs the proportion is considerably higher, and this strongly suggests that a natural infection by some member of the pneumococcal group is of frequent occurrence. Whatever the explanation may be, the phenomenon is one of practical importance in the present connection, for it involves the preliminary testing of the serum of each animal before it can be used as complement.

The hæmolytic system consisted of sheep's corpuscles with the corresponding bæmolytic serum derived from the rabbit.

As may be inferred from what has already been said, the occurrence of group reactions is very marked.

A sufficient amount of an anti-scarlatinal serum will absorb and fix a certain amount of complement in the presence of an antigen derived from any member of the group. It is only by diminishing the amount of immune serum and increasing the amount of hæmolytic serum and complement that differentiation can be obtained among the closely related members of the group. When, however, the test is pushed sufficiently far to separate the closely related diplococci which occur in normal throats, differences begin to appear among the scarlatinal strains, and these show a tendency to separate into two or more groups.

Sera are now being prepared to represent these sub-groups by the immunisation of animals with single strains of the Diplococcus scarlatino, and when these sera are available it should be possible to classify more accurately the throat diplococci and to determine what percentage of them (if any) inust be classed as scarlatinal.

In addition to the eight diplococci from control throats, four other bile-soluble raffinose fermenting pneumococci were examined. Two of these, from cases of pneumonia, have been referred to in connection with their reaction to the monkey. As already stated, one of them 
(Pn. 40) belongs to a type widely separated from the Diplococcus scarlatine, the other (Pn. R) shows a somewhat close relationship to it, but can be distinguished without difficulty. The other two, which were isolated from the pleural cavity, occupy an intermediate position, being separated from the scarlatinal diplococcus with comparative ease. These four pneumococci agree in fermentation reactions with the Diplococeus scarlatince, but their colonies on blood-agar plates do not show the characters which are typical of the latter.

\section{Complement Fixation by the Sera of Scarlet Fever Patients.}

After what has been said regarding group reactions, it is readily intelligible that complement fixation tests with patient's sera are difficult to interpret. In nearly every case of scarlet fever complement fixation with the Diplococcus scarlatinoe can be obtained, but it is also found in about balf the control sera. It is difficult to make this test accurately quantitative. If, however, an arbitrary limit be fixed, e.g., the fixation of one complete hæmolytic dose by $0 \cdot 1$ c.c. of the patient's serum, and this limit be adhered to as closely as the circumstances allow, the cases so far examined may be classed as follows :-

\begin{tabular}{|c|c|c|c|}
\hline \multicolumn{2}{|c|}{ Scarlet Fever. } & \multicolumn{2}{|c|}{ Controls. } \\
\hline Positive. & Negative. & Positive. & Negative. \\
\hline 12 & 6 & 2 & 12 \\
\hline
\end{tabular}

These results afford confirmatory evidence of the causal relationship of the diplococcus to scarlet fever, but they do not appear likely to lead to any useful method of serological diagnosis.

\section{General Conclusions.}

The evidence for and against the view that the Diplococcus scarlatince is the causal infective agent in scarlet fever may now be reviewed.

The diplococcus can be demonstrated in the throats of scarlet fever patients during the first week of the disease in 87 per cent. of the cases, and it disappears as a rule from the throat about the fifth week of convalescence, persisting in an as yet undetermined percentage of cases for a much longer period.

The diplococcus produces in monkeys a disease which in many respects resembles scarlet fever. The most striking point of resemblance is the characteristic change in the polymorpho-nuclear leucocytes. The local tissue necrosis near the site of injection corresponds to the sloughing of the fauces and of the tissues of the neck in the 
so-called septic cases of scarlet fever. Further, the diplococcus has little tendency to produce septicæmia in the monkey, but causes a profound toxæmia, and leads readily to the invasion of the blood stream by other cocci.

The tendency to septicæmia is apparently even less in man than in the monkey. There is as yet no evidence that the diplococcus enters the blood stream in man to any appreciable extent. Even in fulminant cases the blood has usually been found to be sterile; I have myself had no opportunity of examining such cases. As in the case of diphtheria, the Diplococcus scarlatino is confined to the region of primary invasion, which is usually the throat, and there produces toxins which are poured into the blood stream.

Another feature common to the disease in man and in the monkey is scarlatinal rheumatism.

No definite evidence of the occurrence of nephritis in the monkey has yet been obtained.

The most outstanding clinical feature of scarlet fever, namely, the rash, has not been reproduced in the monkey. In this connection it has to be observed that the rash (or subsequent desquamation) is almost the sole clinical criterion on which a diagnosis of scarlet fever can be founded. Only where an angina without rash can be very closely connected with another typical case of scarlet fever is the diagnosis scarlatina sine exanthemate permissible. This aspect of the question may be entirely altered when the causal infective agent in the disease is known, and the percentage of human cases which react without any rash may be much larger than is at present supposed to be the case. It is of course possible to take the attitude that the monkey is insusceptible to scarlet fever, and that the reaction described is not due to the scarlet fever virus, but to an accompanying diplococcus, which no doubt also plays a part in the symptomatology of the human disease. This sceptical attitude was justifiable towards the streptococcal theory of scarlet fever. Not one of the symptoms of scarlet fever (except a transient rise of temperature) can be produced in the monkey by inoculation with streptococci, and no valid distinguishing characters can be shown between the scarlatinal and the ubiquitous pyogenic streptococci.

The theory that scarlet fever is due to an ultra-microscopic or "filter-passing" virus has been recently widely accepted on very insufficient grounds; apparently it rests only on a few inconclusive experiments of Bernhardt's $\left(1911^{1}\right.$ ) which that author was himself unable to repeat $\left(1913^{2}\right)$.

Additional evidence in favour of the diplococcal theory is found in the fact that complement fixation reactions occur with much greater frequency, and with greater intensity in scarlet fever than in control cases. In one case this reaction was found to be distinctly positive on the third day of the disease, while on subsequent examinations on the 
tenth and seventeenth days it could not be detected. This observation is very much against the view that the diplococcus is a secondary invader.

Finally, the biological characters of the diplococcus are not inconsistent with known facts in the epidemiology of scarlet fever. This will be discussed later.

The occurrence in a certain proportion of normal and other control throats (about 34 per cent.) of diplococci almost indistinguishable from the Diplococcus scarlatino undoubtedly makes the demonstration of the causal relationship of the latter to scarlet fever more difficult, but does not in itself invalidate the bypothesis.

Further research will no doubt clear up the relationship of these diplococci, and in the meantime there is little to be gained by speculation as to their possible significance. But it may be permissible to point out that the known epidemiological facts are not inconsistent with the frequent occurrence of scarlet fever "carriers," and to suggest that if these doubtful diplococci should prove to be scarlatinal, they may have generally lost their infectivity for man, but may be capable of regaining infective power under certain conditions. One of these may be passage through the mouse, and the possibility of this occurring under natural conditions is indicated by the feeding experiments on wild mice mentioned above. It is possible that another condition for the acquirement of infectivity is given by infection of the scarlatinal carrier with diphtheria, and if a converse relationship holds between the two diseases this would go some length towards explaining their frequent association with one another.

If the above theory of scarlet fever be finally substantiated, it must be recognised that the group of diplococci, commonly known as "pneumococci," contains a number of species of which two have been associated with definite diseases, namely, Diplococcus pneumonice and Diplococcus scarlatina, the short name of the former being loosely used as a name for the whole group. The so-called "pneumococci" of the tonsils are not normal inhabitants of the mouth, but are, at least potentially, highly pathogenic, and are associated not chiefly with pneumonia, but with a wholly different group of diseases.

It is quite possible that there are in the pneumococcal group many distinct species associated with different diseases, and that in time methods will become available for their distinction. The suggestion may be hazarded that the milk epidemics of septic sore throat which have been described as due to pneumococci will form one such disease.

It is solely the occurrence of an erythematous rash in scarlet fever which has led to its separation clinically as a disease. Further investigation is necessary to show whether this separation can be maintained from the bacteriological standpoint and whether further subdivision may be required.

It has already been shown by various workers that the pneumo- 
cocci found in pneumonia can be divided into at least three types, and here also further investigation may show the necessity for subdivision in a bacteriological sense, which may not run parallel with clinical distinctions. These problems will only be capable of solution when our methods of dealing with the pneumococcal group have reached a state of improvement which renders them comparable with those available in the coli-typhoid group of bacilli.

In conclusion, we may consider shortly how the characters and distribution of the Diplococcus scarlatinoe correspond to the epidemiology of scarlet fever.

For the epidemiological facts a recent paper by Ewart $\left(1916^{3}\right)$ will be followed.

Discussing the disappointing results of hospital isolation in modifying the prevalence of the disease, Ewart concludes that there must be some source of infection such as is hardly influenced by the isolation of known cases of scarlet fever. "This may arise," he says, "through-

"(1) A large number being missed.

"(2) A large proportion reacting atypically

"(3) Infection persisting in certain cases for a considerable period of time.

"(4) Infection arising through other channels than personal contact."

We may take the statistical conclusions as to each of these possibilities and consider to what extent, if at all, they would have to be modified if the Diplococcus scarlatino is accepted as the causal infective agent.

(1) Ewart finds statistical evidence of a large error of omission. The nature of the infective agent is not here involved.

(2) The second suggested cause is considered by Ewart to be a probable factor, and evidence is quoted to show that outbreaks of scarlet fever are often either preceded by or accompanied by a number of cases of sore throat.

We have already seen reason to suggest that possibly many cases of "pneumococcal" angina are in reality atypical reactions to the Diplococcus scarlatino.

(3) With regard to the third factor, Ewart gives reasons for believing that the usual 3 to 4 per cent. of "return cases" does not really represent the percentage of cases which leave hospital in an infective condition, but that the actual figure for such cases is more like 25 per cent.

This figure, reached on purely statistical grounds, may be compared with the percentage of cases (22 per cent.) in which it has already been shown that the Diplococcus scarlatino persists beyond the eighth week of the disease. 
(4) Ewart considers that the first three factors are sufficient in themselves to explain the endemic character of the disease, but in connection with the fourth suggested factor he discusses at some length the question of milk epidemics, concluding that we are hardly justified in regarding milk as a frequent source of infection. His conclusions as to the viability of the hypothetical virus of scarlet fever in milk so exactly correspond with the actual facts regarding the Diplococcus scarlatince that I quote them in full.

"In thirty-seven of the epidemics, in which the evidence seems good, there was contact with a previous case, either at the milking or shortly afterwards. This seems to suggest that the time when milk will absorb the infection is from the time of milking to a few hours afterwards, that is, whilst the milk is warm and comparatively sterile. Instances of contamination of milk with scarlet fever infection in retail shops with no traceable cases are of frequent occurrence in any district. It would thus appear that when milk is cold and the lactic fermentation well advanced, it is apparently not a suitable medium."

We bave already seen that the diplococcus grows readily in sterile milk at temperatures between $20^{\circ}$ and $37^{\circ} \mathrm{C}$. $\left(70^{\circ}\right.$ to $98^{\circ} \mathrm{F}$.), but that in impure cultures in sugar-containing media the acid production inhibits its growth.

Under this heading the possibility of scarlet fever existing as an epizoötic in mice must be again mentioned. Dr. Brownlee has for many years taught that this is one possible explanation of the epidemiology of scarlet fever.

Ewart concludes this part of his argument with the following statement: "From the evidence that has been gathered we can conclude, firstly, that the main factors concerned in the maintenance of the disease are $(a)$ atypical cases, $(b)$ missed cases, $(c)$ persistence of infection (carriers) in a certain proportion; and secondly, that in so far as isolation of known cases does not materially influence the chance of infection outside the home, the amount of floating infection must be extremely large. All must in the ordinary course of events come into frequent, almost daily, contact with one or other of the above classes. Hence we must assume that susceptibility is a much greater factor in reaction to the presence of this parasite, than chance of infection."

This statement would probably be accepted, in its main outlines, by most epidemiologists, and it would therefore appear that, even if further research makes it necessary to identify with one or other sub-group of the Diplococcus scarlatine all the bile-soluble diplococci found in normal throats, no valid objection could be made for that reason, on statistical grounds, to the diplococcal theory of scarlet fever. 


\section{REFERENCES.}

1. Bernhardt, G. . . . . Deutsche med. Wchnschr., Leipzig, 1911, Bd. xxxvii. S. 791.

2.

Ergebn. d. inn. Med. und Kinderheilk., Berlin, 1913, Bd. x. S. 358.

3. Ewart, R. J. . . . . Journ. of Hyg., Cambridge, 1916, vol. xv. p. 208.

4. Matr, W. . . . . Journ. Path. and Bacteriol., Cambridge, 1915, vol. xix. p. 443.

5. Neufeld, F. . . . . Kolle u. Wasserman's "Handbuch d. path. Mikro-organismen," Jena, 1912, Bd. iv. S. 525.

6. Ruediger, G. F. . . Journ. Infect. Dis., Chicago, 1906, vol. iii. p. 755 . 